\title{
O USO DE TECNOLOGIAS DA INFORMAÇÃO E COMUNICAÇÃO EM ÁREAS RURAIS É SUFICIENTE PARA A EDUCAÇÃO CONTINUADA?
}

\author{
Use of Information and Communication Technologies \\ in Rural Areas Enough for Continuing Education?
}

\begin{abstract}
Magda Moura de Almeida1; Cláudio Alves de Albuquerque²; Victor Rezende Veras³; Saulo Herculano de Carvalho4; Ivana Daniela César ${ }^{5}$; Luisa Patrícia Fogarolli de Carvalho6
\end{abstract}

Resumo Objetivos: Esta pesquisa teve o intuito de avaliar o uso das tecnologias de informação e comunicação (TIC) por profissionais de saúde do interior do Ceará. Materiais e Métodos: Estudo transversal, no qual foram aplicados questionários para profissionais de nível superior da Estratégia de Saúde da Família de quatro municípios cearenses vinculados a uma universidade privada pelo internato rural. Resultados: Uma amostra de 72 enfermeiros, dentistas e médicos responderam o formulário. A maioria dos profissionais utilizam diferentes recursos tecnológicos para uso pessoal e para atividades profissionais. O telefone celular é a forma mais comum de TIC utilizada na prática profissional (61\%). O uso recreacional da Internet (96\%) é maior do que como parte da prática clínica diária (37\%), assim como o e-mail (90\% versus $38 \%$ ). Apenas alguns (17\%) têm acesso à Internet no local de trabalho e a maioria (60\%) precisa viajar pelo menos 30 minutos para ter acesso à Internet. Daqueles que têm acesso à Internet no local de trabalho, 91\% utilizam para atividades de autoaprendizagem ou de ensino. Conclusão: Nossos dados apoiam a ideia de que somente o acesso aos recursos tecnológicos não é suficiente. Os dispositivos móveis parecem, no momento, oferecer vantagem de acessibilidade para este grupo.

Palavras-chave: Telessaúde; Saúde da População Rural; Gestão de Ciência, Tecnologia e Inovação em Saúde

Abstract Aims: This study aimed to evaluate the use of information and communication technologies (ICT) for health professionals. Materials and Methods: It was a cross-sectional study in which questionnaires were applied to health professionals of Family Health Strategy of four municipalities of Ceará linked to a private university by rural internship. Results: A sample of 72 nurses, dentists and doctors answered the form. Most professionals use different technological resources for personal and professional activities. The mobile phone is the most common form of ICT used in professional practice (61\%). The recreational use of the Internet (96\%) is higher than as part of daily clinical practice (37\%), either email (90\% versus $38 \%$ ). Only a few (17\%) have Internet access at work and the majority (60\%) have to travel at least 30 minutes to have Internet access. Those who have Internet access at work , 91 \% use it for self-learning and teaching activities. Conclusion: Our data support the idea that only the access to technological resources is not enough. Mobile devices seem to offer advantage of accessibility for this group.

Keywords: Telemedicine; Rural Health; Health Sciences, Technology and Innovation Management

\footnotetext{
1. Mestre em Saúde Pública, Doutoranda de Ensino em Saúde Universidade Estadual de Campinas, Professora Assistente Universidade de Fortaleza - UNIFOR/ Ceará. 2. Médico Graduado, Monitoria Internato de Saúde Coletiva - Universidade de Fortaleza, Fortaleza, Ceará, Brasil; 3. Graduando em Medicina, Monitoria Internato de Saúde Coletiva - Universidade de Fortaleza, Fortaleza, Ceará, Brasil; 4. Graduando em Medicina,- Universidade de Fortaleza, Fortaleza, Ceará, Brasil; 5. Mestre em Saúde Coletiva, Doutoranda em Saúde Coletiva - Universidade Estadual de Campinas; 6. Mestrado em Doenças Infecciosas e Parasitárias, Doutora em Ciências Médicas, Professora Universidade José do Rosário Vellano - UNIFENAS/Minas Gerais
} 


\section{Introdução}

Sargeant ${ }^{1}$ em revisão bibliográfica nas bases de dados do PubMed-Medline, PubMed-Central e do ERIC, de 1996 a 2005, sobre o uso de Tecnologias da Informação e Comunicação (TICs) no ensino médico, categorizou as mesmas em quatro grandes grupos com as seguintes características:

\section{O telefone;}

\section{A tecnologia dos computadores e software;}

3. A vídeo-conferência que incorporou a tecnologia do computador para fornecer interação síncrona, com transmissão de áudio e vídeo, de arquivos, gráficos e etc.

4. As tecnologias portáteis, que podem ser usadas sozinhas ou conectadas à Internet para fornecer informações de suporte.

Não há no DeCS, na BVS-APS ou no MeSH Terms do PUBMED a definição para a palavra "telessaúde". Contudo, "Telessaúde" (TS) e "Telemedicina" são termos equivalentes no panorama brasileiro. Podem ser especificados através de dois eixos importantes com ênfase no suporte assistencial aos profissionais de saúde (teleassistência) ou com destaque para a educação a distância (teleducação) ${ }^{2}$.

Recentemente ampliado pela portaria MS no 2546, de 27 de outubro de $2011^{3}$, o agora Programa Nacional Telessaúde Brasil Redes (TBR) interliga mais de 1.500 Unidades Básicas de Saúde em 11 estados brasileiros, estando 102 desses pontos em municípios cearenses. O projeto tem por missão fornecer, aos profissionais e trabalhadores das Redes de Atenção à Saúde do SUS, os serviços de:

- Teleconsultoria: consulta registrada e realizada entre trabalhadores, profissionais e gestores da área de saúde, por meio de instrumentos de telecomuni- cação bidirecional, com o fim de esclarecer dúvidas sobre procedimentos clínicos, ações de saúde e questões relativas ao processo de trabalho, podendo ser de dois tipos: síncrona - teleconsultoria realizada em tempo real, geralmente por chat, web ou videoconferência; ou assíncrona - teleconsultoria realizada por meio de mensagens offline;

- Telediagnóstico: serviço autônomo que utiliza as tecnologias da informação e comunicação para realizar serviços de apoio ao diagnóstico através de distância e temporal;

- Segunda Opinião Formativa: resposta sistematizada, construída com base em revisão bibliográfica, nas melhores evidências científicas e clínicas e no papel ordenador da atenção básica à saúde, a perguntas originadas das teleconsultorias, e selecionadas a partir de critérios de relevância e pertinência em relação às diretrizes do SUS;

- Teleducação: conferências, aulas e cursos, ministrados por meio da utilização das tecnologias de informação e comunicação.

O funcionamento da rede envolve a atuação de Núcleos Técnico-Científicos de TS, que são instituições responsáveis pelo fornecimento dos serviços de saúde a distância previstos no projeto e Pontos de TS, que são as unidades de atenção à saúde de onde os profissionais do SUS demandam estes serviços.

A gestão do Telessaúde Brasil Redes é composta por uma Coordenaação Nacional, exercida pelo Ministério da Saúde, Coordenações Estaduais exercidas pelas Secretarias de Saúde dos estados envolvidos, Comitês Gestores Estaduais e Núcleos Técnico-Científicos de Telessaúde, com funções e composição detalhados na portaria.

O projeto vem sendo mantido com recursos do Ministério da Saúde desde o início de sua fase piloto, em 2007, quando foi previsto o investimento de 
R\$15.000.000,00 para a implantação dos primeiros 900 pontos, distribuídos por nove estados, incluindo o Ceará. De acordo com dados do Portal da Transparência do Governo Federal ${ }^{4,5}$, já foram repassados ao estado R\$ 2.450.000,00 relacionados ao Projeto Telessaúde Brasil.

$\mathrm{Na}$ etapa piloto, cada município recebeu equipamentos de informática financiados pelo Ministério da Saúde (computador desktop, impressora multifuncional e webcam). $\mathrm{O}$ acesso à internet em banda larga era contrapartida municipal ${ }^{3}$.

O financiamento da atual fase de expansão permanece a cargo da esfera federal e obedece à regulação do Programa de Requalificação das Unidades Básicas de Saúde ${ }^{5}$, que prevê investimentos anuais de até $R \$ 3.550 .000,00$ para projetos que contemplem mais de 900 equipes da Estratégia de Saúde da Família. Uma vez implementado no estado, as três esferas de governo serão responsáveis por garantir a sustentabilidade técnico-financeira do Programa. Só em 2011, o Ceará recebeu a quantia de R\$ 525.000,00 dos recursos do Fundo Nacional de Saúde, referente ao Programa de Requalificação de UBS- Informatização e Telessaúde.

Apesar do grande investimento, o conhecimento disponível sobre a contribuição das TICs - e especificamente do programa nacional no suporte a profissionais da Atenção Primária a Saúde (APS) - é incipiente.

Sabe-se que os serviços se diferenciam na sua capacidade tecnológica e de infraestrutura, o que proporciona graus de respostas diferenciadas aos problemas de saúde e aos processos de educação permanente dos profissionais associados. Portanto, se carece de estudos que considerem os diferentes cenários e contextos em um país de dimensões continentais.

Filho $^{2}$, em tese de doutorado, avalia o uso da TS como apoio para médicos da APS no Rio Grande do Sul e reflete: “É fundamental saber não apenas se um tipo de intervenção é efetiva, mas quando, para quem e com quais recursos ela é efetiva."
A hipótese deste estudo era de que existe subutilização dos recursos tecnológicos disponíveis em zonas rurais, de modo a não promover o efeito esperado pelo Projeto Telessaúde Brasil Redes, de qualificação da APS e diminuição do número de encaminhamentos desnecessários.

Para esclarecer esse questionamento avaliou-se o modo de uso das tecnologias de informação e comunicação (TICS) para a resolução dos problemas de saúde locais e para a educação permanente dos profissionais de saúde em uma amostra de municípios do interior do Ceará.

\section{Materiais e Métodos}

Tratou-se de um estudo transversal, exploratório e descritivo, realizado a partir da coleta de dados com formulários estruturados, no período de janeiro de 2012 a janeiro de 2013 nos municípios de Aratuba, Camocim, Carnaubal e Tabuleiro do Norte, distantes respectivamente $128 \mathrm{~km}, 370 \mathrm{~km}, 361 \mathrm{~km}$ e $222 \mathrm{~km}$ da capital.

A amostra foi intencional, com profissionais de nível superior da Estratégia de Saúde da Família (ESF) destes quatro municípios vinculados ao estágio rural de uma universidade particular. Nem todos os municípios eram vinculados ao TBR, sendo este dado utilizado para realizar a comparação entre grupos de profissionais com acesso a diferentes tecnologias.

A estratégia de coleta de dados de dados envolveu pelo menos duas visitas a cada município, sendo a primeira uma reunião com a gestão municipal para a apresentação do projeto.

Em um segundo momento, os questionários foram distribuídos a todas as equipes da ESF para serem preenchidos. Essa distribuição ocorreu de duas maneiras a critério de cada gestor: ou presencial em cada unidade de saúde, ou em reuniões com as equipes nos prédios da administração. Todos os 
participantes assinaram o Termo de Consentimento Livre e Informado.

O projeto foi aprovado no Comitê de Ética na Universidade de Fortaleza sob o CAAE 05751312.5.0000.5052.

As respostas aos formulários autoaplicáveis formaram um banco de dados no software Excel. A análise de dados foi realizada no software estatístico SPSS ${ }^{\circledR} 16.0$ for Windows.

Para análise bivariada de variáveis categóricas foi aplicado o"Teste Qui-Quadrado" e a "Análise dos Resíduos". O resíduo ajustado tem distribuição normal com média zero e desvio padrão igual a 1. Desta forma, caso o resíduo ajustado seja maior que 1,96, em valor absoluto, pode-se dizer que há evidências de associação significante entre as duas categorias. Quanto maior for o resíduo ajustado, maior a associação entre as categorias.

Para medir o grau de correlação entre duas variáveis quantitativas foi calculado o Coeficiente de Correlação de Pearson. O coeficiente de Correlação Linear de Pearson ( $\mathrm{r}$ ) é uma medida que varia de $-1 \mathrm{a}+1$. O coeficiente fornece informação do tipo de associação das variáveis através do sinal:

- Se $\mathbf{r}$ for positivo, existe uma relação direta entre as variáveis (valores altos de uma variável correspondem a valores altos de outra variável);
- Se $\mathbf{r}$ for negativo, existe uma relação inversa entre as variáveis (valores altos de uma variável correspondem a valores baixos de outra variável);

- Se $\mathbf{r}$ for nulo ou aproximadamente nulo, significa que não existe correlação linear.

Os resultados foram organizados em forma de tabelas e gráficos sendo expressos em média, frequências absoluta e relativa.

\section{Resultados}

Os 72 respondentes estavam distribuídos da seguinte maneira: 13 profissionais de saúde de Aratuba $(18,1 \%)$, 12 de Carnaubal $(16,7 \%)$ e 11 de Tabuleiro do Norte (15,3\%), todos esses municípios com TBR. O controle foi feito com o município de Camocim, sem TBR, que respondeu com $50 \%$ da amostra, ou seja, 36 profissionais.

Na Tabela 1 estão descritos os principais dados sociodemográficos da população estudada. Com exceção da categoria médica, identifica-se a feminilização importante dos profissionais de saúde vinculados a ESF no interior do Ceará, principalmente na enfermagem.

Os médicos são os profissionais com a maior média de idade (36,5 \pm anos) e de tempo de atuação profissional

\begin{tabular}{|l|l|c|c|}
\hline PROFISSÃO & & Média & Desvio Padrão \\
\hline \multirow{2}{*}{$\begin{array}{l}\text { DENTISTA } \\
\text { Feminino 57\% }\end{array}$} & Idade & 29,3 & 3,7 \\
\cline { 2 - 4 } & Tempo na ESF (meses) & 38,0 & 32,5 \\
\cline { 2 - 4 } $\begin{array}{l}\text { ENFERMEIRO } \\
\text { Feminino 71\% }\end{array}$ & Tempo de atuação profissional (anos) & 6,9 & 3,9 \\
\cline { 2 - 4 } Masculino 29\% & Idade & 33,3 & 8,8 \\
\cline { 2 - 4 } MÉDICO & Tempo de atuação profissional (anos) & 57,6 & 51,1 \\
Feminino 38\% & Idade & 8,3 & 15,5 \\
\cline { 2 - 4 } Masculino 62\% & Tempo na ESF (meses) & 36,5 & 82,6 \\
\cline { 2 - 4 } & Tempo de atuação profissional (anos) & 49,2 & 15,1 \\
\hline
\end{tabular}

Tabela 1. Perfil demográfico dos profissionais de nível superior da ESF nos municípios de Aratuba, Camocim, Carnaubal e Tabuleiro do Norte, 2012 
(12,4 15 anos). Os enfermeiros, contudo, possuem a maior média (4,8 \pm 4 anos) de tempo de trabalho na ESF.

Houve associação significativa entre a idade dos sujeitos e o tempo de atividade na ESF com enfermeiros e médicos (Tabela 2). O cálculo do coeficiente de Correlação Linear de Pearson ( $r$ ) foi positivo em ambas as categorias.

O tempo de formatura e o tempo de trabalho na ESF também demonstrou correlação linear em todas as categorias profissionais (Tabela 2). Ou seja, quanto maior o tempo que o profissional encontra-se atuando na ESF, mais anos de formado ele possui.

A maioria dos profissionais qualifica como "importante" e "muito importante" o uso das TICs na condução dos problemas de saúde dos seus pacientes $(41,7 \%$ e $52,8 \%$ respectivamente), assim como para sua própria atualização e educação continuada (25\% e 72,2\%).
Entretanto, quando questionados se as TICs ofertadas pelo município ajudavam a qualificar os serviços de saúde no seu município, 18\% não concordaram, 43\% concordaram parcialmente e 31\% concordaram plenamente.

No Gráfico 1 são identificadas as TICs utilizadas pelos sujeitos do estudo em atividades profissionais e de lazer/recreacionais. Todas elas são mais utilizadas para uso pessoal do que para uso profissional. A internet é a TIC mais utilizada para atividades privadas (95,8\%), no entanto para atividades profissionais só é utilizada por $37,5 \%$.

Não houve diferença em relação a utilização das TICS para uso privado entre os sexos. Todavia os homens utilizam mais computadores de mesa para o trabalho $(p=0,01$ e resíduo ajustado $=2,4)$.

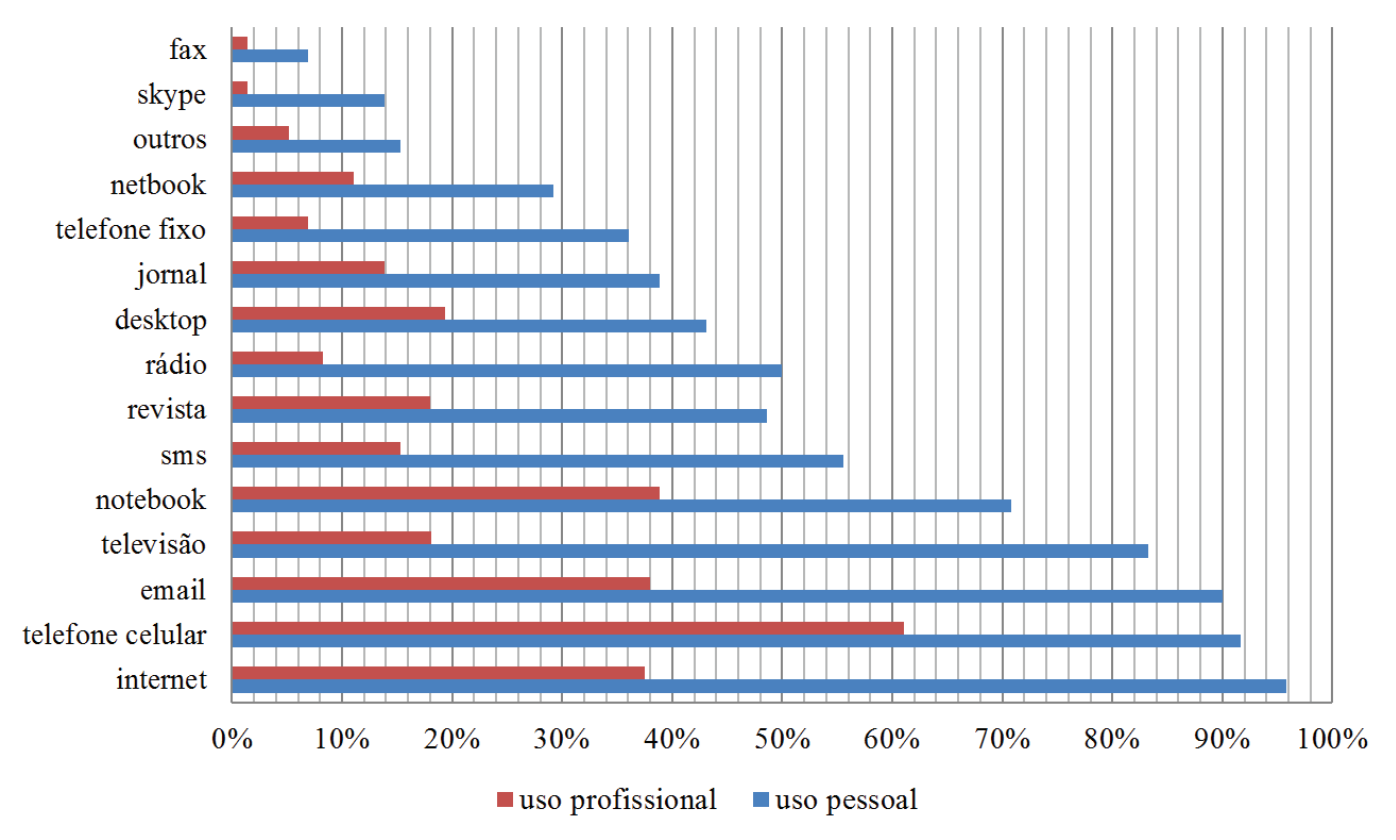

Gráfico 1. Distribuição do uso de TICs por profissionais de saúde em áreas rurais, $2012(\mathrm{n}=72)$.

\begin{tabular}{|c|c|c|c|c|c|c|}
\hline & \multicolumn{6}{|c|}{ Tempo de Trabalho na ESF } \\
\hline & \multicolumn{2}{|c|}{ Dentista } & \multicolumn{2}{|c|}{ Enfermeiro } & \multicolumn{2}{|c|}{ Médico } \\
\hline & $\mathbf{r}$ & $\mathbf{p}$ & $r$ & $\mathbf{p}$ & $\mathbf{r}$ & p \\
\hline Idade & 0,72 & 0,06 & 0,81 & 0,001 & 0,77 & 0,002 \\
\hline Tempo de Formado & 0,82 & 0,02 & 0,86 & 0,000 & 0,79 & 0,001 \\
\hline
\end{tabular}

Tabela 2. Cálculo de Coeficiente de Pearson entre o tempo de trabalho na ESF, a idade e o tempo de formatura dos profissionais de saúde em zonas rurais do Ceará 


\section{A Internet}

Os médicos são os profissionais que mais utilizam a internet, notebook e e-mail (56,5\%) para o trabalho. Tanto enfermeiros quanto médicos apresentaram relação estatisticamente significante com $\mathrm{p}<0,005$ ao "teste do Qui Quadrado", com resíduo ajustado de 3,1 para o uso de profissional da internet e e-mail, que também se encontra intrinsicamente ligado a faixa etária menor de 25 anos ( $p=0,003$ e 3,4 de resíduo ajustado).

Não existe associação do uso da internet com a utilização de computadores de mesa. Para os médicos, o uso da internet para o trabalho está fortemente associado a aquisição de um notebook, também com $\mathrm{p}<0,005$ e resíduo ajustado de 3,1.

Não foi averiguada associação estatística entre o uso profissional da internet e atividades de teleducação (conferências, aulas, cursos) ou solicitação de recursos do TBR como teledermatologia, tele-ECG ou segunda opinião formativa.

Como seria de se esperar, os municípios com TBR, possuem maior utilização dos recursos oferecidos, principalmente a teledermatologia e o tele-ECG. Contudo, não houve diferença em relação ao uso de teleducação ou de segunda opinião formativa entre os municípios com ou sem TBR. E mesmo, nas localidades conveniadas ao TBR ainda é grande percentual de profissionais que não faz uso das suas ferramentas, como pode ser verificado na Tabela 3.

A faixa etária acima dos 35 anos é a que menos utiliza a teledermatologia ( $p=0,05$ e resíduo ajustado de 2,7 ), o
tele-ECG ( $p=0,03$ e resíduo ajustado de 2,7) e a segunda opinião formativa ( $p=0,04$ e resíduo ajustado de 2,3 ).

\section{O Telefone Celular}

O telefone móvel por sua vez é a TIC mais utilizada $(61,1 \%)$ para o trabalho dos profissionais de saúde na área rural. Os enfermeiros e os médicos são os que mais utilizam essa ferramenta $(65,5 \%$ e $65,2 \%)$ para estes fins.

É relevante destacar que, quando questionados se eram capazes de acessar uma rede de celular diretamente no seu local de trabalho, houve frequência de respostas diferentes entre as categorias. Os dentistas foram os profissionais que referiram ter mais acesso a rede de telefonia móvel no trabalho (60\%), seguidos pelos enfermeiros (42,9\%) e médicos (31,8\%). Grande parte referiu já ter usado a rede de celular para ajudálos com autoaprendizagem: $66,7 \%$ dos dentistas, $61,5 \%$ dos enfermeiros e $60 \%$ dos médicos.

A época do envio do projeto ao Comitê de Ética, o uso de tablets ou smartphones não era tão difundido como atualmente, e consequentemente não foram inseridos nominalmente na lista das TICs pesquisadas, podendo ter sido incluídos na categoria "outros" presente no formulário.

Para averiguar se o uso dos telefones móveis estava associado ao uso de redes de internet e consequentemente, às ferramentas associadas a Web 2.0 e não às características da rede de telefonia, foram avaliadas as associações entre a utilização para o trabalho do telefone celular com o uso da internet, e-mail e SMS (Short Message Service).

\begin{tabular}{|l|c|c|c|}
\hline \multirow{2}{*}{} & \multicolumn{2}{|c|}{ Tempo de Trabalho na ESF } \\
\cline { 2 - 4 } & Utilizam & Não Utilizam & p \\
\hline Tele-educação: conferências, aulas e cursos & 19,4 & 80,6 & 0,234 \\
\hline Tele-dermatologia & 11,1 & 88,9 & 0,003 \\
\hline Tele-ECG & 13,9 & 86,1 & 0,001 \\
\hline Segunda Opinião Formativa & 9,7 & 90,3 & 0,047 \\
\hline
\end{tabular}

Tabela 3. Utilização de recursos do TELESSAÚDE BRASIL REDES (TBR)/UNASUS em municípios conveniados ao TBR no Ceará 
usa celular no local de trabalho para aprendizagem/ensino

rede de celular no local de trabalho

srnet há 30 minutos do local de trabalho

usa internet no local de trabalho para aprendizagem/ensino

internet no local de trabalho

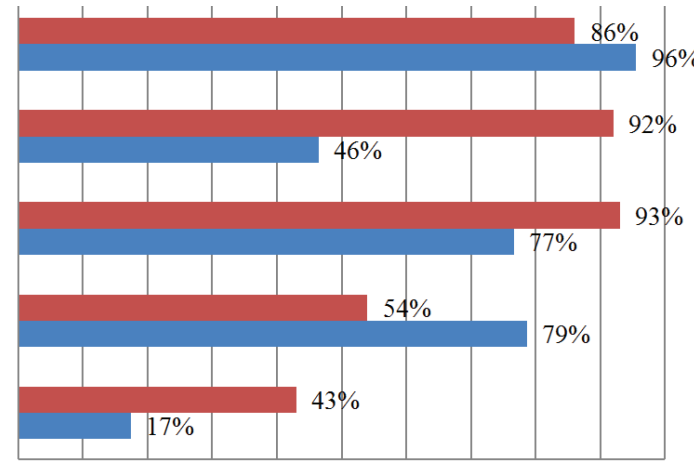

$\begin{array}{llllllllllll}0 \% & 10 \% & 20 \% & 30 \% & 40 \% & 50 \% & 60 \% & 70 \% & 80 \% & 90 \% & 100 \%\end{array}$

•Uganda, 2009 Ceará, 2012

Gráfico 2. Comparação do uso de TICs em zonas rurais da Uganda e Ceará.

O teste do "Qui Quadrado" não apresentou associação para nenhumas das variáveis.

Apesar de todas as categorias referirem utilizar a telefonia móvel em situações da prática do trabalho, este uso não está associado a nenhuma das facilidades associada à Web 2.0. Inferindo que esta utilização se deve eminentemente a ligações telefônicas. Também, não houve associação estatística entre a presença de rede telefônica no trabalho e o uso do telefone, induzindo à suposição de que o uso do telefone para fins profissionais não ocorre necessariamente no próprio ambiente de trabalho.

\section{As Redes Sociais}

O Facebook é a rede social mais acessada pelos dentistas (90\%), enfermeiros (79,3\%) e médicos $(65,2 \%)$. Não utilizam, mas possuem interesse em utilizar 5\%, 13,8\% e $17,4 \%$ das respectivas categorias profissionais supracitadas. Não possuem perfis em redes sociais e continuam sem interesse em tê-los representam respectivamente $5 \%, 6,9 \%$ e $13 \%$ destes grupos.

\section{Discussão}

O profissional de saúde vinculado à ESF em zonas rurais ainda apresenta comportamento diferenciado quando se trata do uso de tecnologia para assuntos privados e profissionais. Aparentemente essa tendência condiz com o status mundial para países em desenvolvimento, que parecem estar ainda no início desse processo de implementar largo acesso a informação em saúde ${ }^{8}$.

A correlação linear entre idade, tempo de atuação profissional e de atividade na ESF infere que esta política vem se consolidando um campo de atuação considerável no mercado de trabalho do médico em zonas rurais nos últimos anos.

O alto desvio padrão dessas variáveis revela importante heterogeneidade deste subgrupo que merece ser considerada e avaliada para que o uso das TICs seja potencializado, como por exemplo, a baixa utilização dos recursos de teleassistência pela faixa etária de 35 anos.

Filho $^{2}$ também identificou maior idade física e profissional, maior tempo de permanência dos médicos na ESF, e consequentemente menor uso de teleconsultorias. Essas peculiaridades devem ser levadas em consideração na formulação de políticas públicas que demandem vultosos investimentos. Será necessário delinear estudos específicos para revelar se o menor uso de TICs por quem tem mais idade se deve a maior experiência acumulada, por menor familiaridade prévia com informática ou por dificuldade de enfrentamento de sua limitação pessoal ${ }^{6}$.

O baixo uso do computador de mesa nas atividades trabalhistas infere, junto aos outros dados analisados que os recursos aplicados na aquisição de equipamentos no TBR podem estar sendo subutilizados, já que a base de ligação entre o profissional de saúde e o programa devia ser inicialmente, um desktop dentro na unidade básica de saúde.

Se compararmos os dados coletados com pesquisa realizada em Uganda em $2009^{7}$ podemos perceber que os profissionais da zona rural do país africano possuem maior acessibilidade a rede de internet e de telefonia móvel que os profissionais no Brasil (Gráfico 2). 
Concomitante, os mesmos dados demonstram a potencialidade destas ferramentas, pois, apesar do acesso inferior ao dos africanos, os profissionais de saúde brasileiros utilizam mais a internet e o telefone celular para o autoaprendizado. O melhor acesso à informação através dos recursos de telessaúde está intimamente relacionado com a atitude do indivíduo em procurar informações a partir da internet e/ou de outras fontes para responder a perguntas específicas ${ }^{8}$.

Este fato condiz com a percepção destes trabalhadores sobre a importância das TICs tanto para a educação continuada quanto para a condução dos problemas de saúde da comunidade que assistem.

O TBR pretende qualificar os profissionais da APS através do uso de TICs, acreditando que, deste modo, possa aprimorar a qualidade do serviço de saúde ofertado e sua resolutividade, diminuindo o número de encaminhamentos desnecessários.

Esse pressuposto encontra dados que o fortalecem em estudo como o de Oliveira e Gonçalves ${ }^{9}$ que identificaram diminuição de 16,7\% de encaminhamentos da APS ao otorrinolaringologista, após três tipos de intervenções: videoaulas, teleconsultoria com especialista e videoaulas/teleconsultoria. Contudo, a mesma pesquisa ressalta que houve baixa adesão à teleconsultoria (média de 0,33/participante), que não apresentou efeito na melhora do conhecimento teórico dos participantes, quando comparada com a videoaula - um indicativo da baixa efetividade deste método tanto para a teleassistência com para a teleducação. A videoaula agregou conhecimento teórico quanto ao teste imediato, mas não mudou a prática profissional do médico.

A limitação do tamanho amostral deste estudo não permite generalizar e extrapolar seus resultados, mas nossos dados reforçam o cenário supracitado quando demonstram comportamentos equivalentes entre os municípios com e sem TBR. Evidente que nenhum benefício pode acontecer se o serviço não for aces- sado, contudo a disponibilidade das TICs parece não ser preditor para o seu uso.

A discrepância de acesso à rede de telefonia móvel entre as categorias profissionais dentro uma mesma equipe de ESF pode ser explicada pelo fato de que os dentistas usualmente permanecem dentro de uma única UBS, na área urbana dos municípios. Já os enfermeiros e médicos realizam deslocamentos frequentes para consultas e visitas domiciliares em pontos remotos da mesma cidade.

Apesar disso, considerando que o telefone celular foi identificado como a TIC de maior uso profissional e que não houve associação entre a presença de rede telefônica no trabalho e o seu uso, podemos inferir que os sujeitos avaliados utilizam esta TIC para atividades profissionais, mas fora do seu ambiente de trabalho. Ou seja, o médico ou enfermeiro, mesmo realizando atendimentos em pontos remotos, fazem uso profissional do celular quando retornam aos pontos de apoio principais.

\section{Conclusões}

A qualificação da ESF através de políticas inovadoras, com o uso de novas tecnologias, é possível, mas é necessário realizar avaliações sistemáticas para que seus recursos sejam constantemente adaptados e bem direcionados.

Nossa exploração não identificou somente fragilidades, mas permitiu apontar outros caminhos que podem ser seguidos. Por exemplo, a adoção de tecnologias como o telefone móvel, tecnologia mais antiga, mais simples e mais barata, como outras que acabaram tornando-se negligenciadas, em detrimento de equipamentos mais modernos, mais onerosos e ainda menos utilizados no cenário dos profissionais de saúde em áreas rurais, quiçá por limitações técnicas ou por falta de estímulo. 


\section{Referências}

1. Sargeant JM. Medical education for rural areas: Opportunities and challenges for information and communications technologies. J Postgrad Med [serial online] 2005 [cited 2012 Mar 18];51:301-7. [http://www.jpgmonline.com/text.asp?2005/51/4/301/19244]

2. Filho EDC. Telessaúde em apoio à Atenção Primária à Saúde no Brasil. Revista Brasileira de Medicina de Família e Comunidade, [S.I.], v. 3, n. 11, p. 210-215, Nov. 2010. ISSN 2179-7994. Disponível em: http://www.rbmfc.org.br/rbmfc/article/view/227>. Acesso em: 06 Dez. 2013. doi:10.5712/rbmfc3(11)227.

3. Ministério da Saúde. Portaria n 2.546, de 27 de outubro de 2011. Redefine e amplia o Programa Telessaúde Brasil, que passa a ser denominado Programa Nacional Telessaúde Brasil Redes (Telessaúde Brasil Redes). Brasil, Diário Oficial União. 28 out 2011; N. 208; Seção 1; p. 50-51.

4 Portal da Transparência. Detalhes do Convênio: 667899. Brasil. Controladoria-Geral da União. Acesso em: 8 jul. 2012. [http://www.portaltransparencia.gov.br/convenios/ DetalhaConvenio.asp? CodConvenio $=667899 \&$ TipoConsulta $=0]$.
5. Portal da Transparência. Detalhes do Convênio: 663794 Brasil. Controladoria-Geral da União. Acesso em: 8 jul. 2012 [http://www.portaltransparencia.gov.br/convenios/ DetalhaConvenio.asp?CodConvenio $=663794 \&$ TipoConsulta $=0]$.

6. Lewis T, Synowiec C, Lagamarsino G, Schweitzer J.E-health in low- and middleincome countries: findings from the Center for Health Market Innovations. Bulletin of the World Health Organization 2012;90:332-340. doi: 10.2471/BLT.11.099820\}.

7. Chang LW, Mwanika A, Kaye D, Muhwezi WW, Nabirye RC, Mbalinda S, et al. Information and communication technology and community-based health sciences training in Uganda: perceptions and experiences of educators and students. Inform. Health Soc. Care [Internet]. 2012 Jan [cited 2012 Mar 22];37(1):1-11. Available from: http://www.ncbi.nlm.nih.gov/pubmed/21332303

8. Mugisha JF. Using information and communication technology to revitalize continuing professional development for rural health professionals: evidence from a pilot project. Rural Remote Health. 2009;9(4):1222.

9. Oliveira MH De, Gonçalves DU. Videoaula ou Teleconsultoria no Aprendizado em Otorrinolaringologia do Médico de Família. Rev Bras Educ Med. 36(4):531-5. 